\title{
ARTIFICIAL INTELLIGENCE AND CYC
}

\author{
Ameer Hamza Khan, Amina Zaheer, Syeda Binish Zahra
}

\begin{abstract}
Since 1984, it is enormous work going on for the accomplishing of the project Cyc
\end{abstract} ('Saik'). This work is based on knowledge of "Artificial Intelligence" which is developed by the Cycorpcompany and by Douglas Lenat at MCC. It's a Microelectronics and Computer Technology Corporation (MCC) part for so long. The dominant aim of Cycorp to develop this system is to just clarify anything in semantical determination rather than syntactically determination of words commands by the machine in which Cyc is installed to do some job. The other objective was in the building of Cyc is to codify, in a form which is usable by the machine, where knowledge's millions piece that composes common sense of a normal human or the common sense made in the human brain. Cyc presents a proprietary schema of knowledge representation that utilized first-order relationships. The relationships that presents between first-order logic (FOL) and first-order theory (FOT). After a long time, in1986, Cyc's developer (Douglas Lenat) estimate that the total effort required to complete Cyc project would be 250,000 rules and 350 man-years. In 1994, Cyc Project was the reason behind creating independency into Cycorp, in Austin, Texas. As it is a common phrase that "Every tree is a plant" and "Plants die eventually" so that why by the mean of this some knowledge representing pieces which are in the database are like trees and plants like structures. The engine is known as an inference engine, able to draw the obvious results and answer the questions correctly on asking that whether trees die. The Knowledge Base (KB) system, which is included in Cyc, contains more than one million humans like assertions, rules or commonsense ideas. These ideas, rules, and human-defined assertions are describing or formatted in the language known as CycL. They are based on the predication of calculus and many other human-based sciences, which has syntax similar to that of the language "LISP". Though some extend the work on the Cyc project continues as a "Knowledge Engineering", which represents some facts about the world, and implementing effective mechanisms which are derived after reaching the basic level conclusion on that knowledge. As Cyc include the firstorder logic and first order theory, which exist in some relationship; so it definitely uses and handle some other branches for human-interaction like mathematics, philosophy, and linguistics. However, increasingly, the other aim of Cycorp while developing Cyc is involving an ability, which is given to the Cyc system that it can communicate with end users, by use of 
CycL, processing of natural language, and can assist with the knowledge formation process through the machine learning.

\section{HISTORY}

As there is a great problem which is faced by many alignments and company and that's software's brittleness and it may be with us after some 50 s years. As if a man asks a medical diagnosis, system about his health and about some disease with which he/she is suffering or was suffered in past the machine probably not able to answer your questions accurately even if that system is world class diagnostic expert. Or a car loan system may grant a person for a car loan when he/she entered about job that he/she has 19 years of job experience at the current job even he/she have a date of birth of 1989. So for handling these kind of miserable software's problem, the project Cyc was introduced in Austin, Texas from 1984 by Doug Lenat at MCC, which is a project of Artificial Intelligence, proposing and making this project to enable a computer or a machine to process natural language for example after the completion a machine will become hear, accept, understand human natural language, and give human-like reasoning which will become the most advance part of humancomputer interaction, which is using the method or rules of Microelectronics and computer technology, the first and one of the largest computer industry research and development consortia (the combination of two organizations or companies). The name "Cyc" is a registered trademark owned by Cycorp. As it is a smaller version of the knowledge base but the originally knowledge-base is proprietary by Cycorp to Cyc, which is used to establish a common vocabulary for the purpose of automatic human-like reasoning. Cyc or prelease OpenCyc under an open source license, and recently Cyc system is being made available to AI researchers under a research purpose as ResearchCyc. And it is used in many applications only because of the publishing it in the open sources.

\section{INTRODUCTION}

Today, we have an impressive amount of data to which we interact with and which we use to solve the world's most Challenging problems around us, but what happened when our data is reached to considerable size, unhandled, this stage make the data scientist, traditional analytics and neural artificial intelligence experts worried. This is a project made in the field of Artificial intelligence which is 
made for reading, interpreting, understanding, and giving some suitable output to end-user. So, at this point, human began to think how do we understand data? And how do we translate it into meaningful action? This project is a lot of worth; also it is the next step in the increment of human-man interaction (HMI). After the accomplishing of this project no user has to even touch a machine in future or interact with controls like buttons, joy or giro sticks it's a simple mechanism of talking with a machine to operate it in more efficient manner. MCC doing a lot of work regards to Cyc and also release some of its modules to researchers and to user so that they get some useful work from it and also to enhance it in some manners. The above mentioned-questions are the study since 30 years. From 1981 a small group of computer scientists began to start working on the shared vision and building an intelligent system that uses real-world knowledge and understand and interpreting that for human-like reasoning, so after the millions of dollars and millions of Ph.D. hours, that vision is fully realized. Meet "CYC", Cyc is the artificially intelligent system enterprise which provides humanlike understanding and reasoning to some complex systems and calculates depth, speed and scalability to human taught which is the world's first largest, and smartest knowledge-based system by applying intelligent reasoning and common sense to data acknowledge. Cyc gives you an environment to solve world's most challenging business problems.

"Welcome to future problem-solving. Welcome to the age of intelligent action." Releases:

MCC release the two major sources to the public for their furthermore researchers and for their work and implementation. The two of the module is given below;

\section{OpenCyc:}

In June 2012, the newer version of OpenCyc of 4.0, released. As it is stated before that $\mathrm{Cyc}$ lie on some relations so OpenCyc 4.0 includes the ontology of whole Cyc which contains terms in millions, also with millions of human-like declarations related to the expressions with each other; however, these are mostly taxonomic proclamations, in Cycthe difficult rules aren't available in it. Knowledge-based include 239,000 concepts and 2,093,000 facts and which can be seen on the OpenCyc.org website.

OpenCyc is no longer available in the era of 2017.The OpenCyc's very first version was released in 2002, spring, and contains a considerable amount of concepts and facts which are $\mathbf{6 , 0 0 0}$ concepts and 60,000 facts. The knowledge-based released under the open license. For meeting the 
needs of its users, Cycorp was specified its intentions for releasing OpenCyc under parallel and unhindered licenses. CycL and SubL interpreter (the program for browsing and editing the databases and for drawing inferences as well) released free of cost, but as a dualistic. It is also available for the operating systems like Linux and Microsoft Windows. Texaiproject was released the RDF supported contents which extract from OpenCyc.

\section{ResearchCyc:}

Before 6 months of the releasing date of OpenCyc at July 2006, Cycorp releases the executable of ResearchCyc 1.0 version. This version of $\mathrm{Cyc}$ aimed at the research community, free of cost while the ResearchCyc in the beta stage of its development in the 2004 and then finally it was released to open source for researchers on January 2005.Also the taxonomic information exists in OpenCyc, ResearchCyc rather than syntactical knowledge it involves considerably additional semantic information like more facts about concepts in its knowledge-base system, and comprises a large dictionary, English analyzing and cohort tools, and Java-based user interfaces for knowledge enhancing and enquiring. Moreover, it holds a system for Ontology- based data amalgamation.

\section{Cyc's Applications:}

\section{Knowledge based terrorist detection:}

The knowledge base terrorism detection application or system of Cyc which is under development whose aim is to contains all the information about the "terrorist" groups, their group members also those members who are leading their groups, philosophy, creators, guarantors, associations, services, sites, capitals, competences, purposes, deeds, strategies, and full metaphors of exact terrorist actions. The information is stored as reports in mathematical reasoning, appropriate for computer sympathetic and cognitive.

\section{Cyclopedia:}

Cyclopedia is being developed; it overlays Cyc keywords on sheets occupied from Wikipedia sides.

\section{Cleveland clinic foundation:}

The Cleveland Clinic used Cyc to develop a natural-language interface for biomedical information and queries. The question is construed into a set of CycL (higher-order logic) parts with open variables, then after put on various limitations like medical- 
domain knowledge, human like commonsense, syntax, and many more. There is a way to fit those fragments together to form one semantically expressive official query.

\section{Methodology:}

All the concepts, which are used in Cyc are known as "Constraints". Which are start from an optional syntax of "\#\$" and which are case-sensitive. Many coefficients are:

- \#\$BillClinton or \#\$Frace means individual item known as individuals.

- More than one means, Collections, such as \#\$Tree-ThePlant (containing all trees) or \#\$EquivalenceRelation (including all correspondence relations). A fellow of a collection is called an instance.

- For specific one, which produces new terms are known as Functions. For example, \#\$FruitFn, when providing them an argument which describes a type of plant (s), will give the collection of its fruit. Conventionally, function constants begin with uppercase letter and end at string "Fn".

- Truth Functions, returning of either true or false value which can be applied to one or more other concepts are done by these functions. For example, \#\$siblings are the name of fraternal relationship, which is true if and only if when two arguments that are passed, are siblings. Starting letter of the constants of these functions is lower-case. Truth functions are divided into logical connectives, quantifiers, and predicates.

Important bases are \#\$isa and \#\$genls. Where the first one predicate or base define that one item is an instance of some collection while, on the other hand, the second one describe that one collection is the sub-collection of some other collection. Facts about concepts are declared using sureCycL sentences. Predicates are written before their arguments, in parentheses:

(\#\$isa \#\$BillClinton \#\$UnitedStatesPresident)

"Bill Clinton belongs to the collection of U.S. presidents" and

(\#\$genls \#\$Tree-ThePlant \#\$Plant)

"All trees are plants". 
(\# capitalCity \#\$France \#\$Paris)

"Paris is the capital of France."

Variables are also contained by the sentences, where strings starting with "?" sign are known as "Rules". An important rule declared for"\#\$isa" predicate reads;

(\#\$implies

(\#\$and

(\# isa ?OBJ ?SUBSET)

(\#\$genls ?SUBSET ?SUPERSET))

(\#\$isa ?OBJ ?SUPERSET))

with the interpretation "if OBJ is an instance of the collection SUBSET and SUBSET is a subcollection of SUPERSET, then OBJ is an instance of the collection SUPERSET". Another typical example is

(\# \$relationAllExists \#\$biologicalMother \#\$ChordataPhylum \#\$FemaleAnimal)

It means, every instance of the collection, \#\$ChordataPhylum (i.e. for every chordate), it is a female animal exist, who is its mother which is defined by predicate \#\$biologicalMother.

Knowledge-base is separated into theories

\section{CONCLUSION}

The conclusion of the system is so complex as it is a contentious development it is a considerable big effort to step forward and try to implement whatever this field proposal into a solitary direction. A concentrated effort required for achieving Break through. The problem of that inspire known as "microtheories (Mt)", which is the collection of concepts and fact snaturally relating to a specific kingdom of information. Each "microtheory" has a name describe by unvarying constant; microtheory constants contain the string "Mt" by resolution.

Cyc is the fragility and highly brittleness of programs. It is only the narrow of sustenance behind the programs' embryonic symbols which cause problems in expert systems, knowledge sharing system, natural language processing, and human interfaces. Cyc introduces the way 
to resolve this problem by the mean of the introduction of the deeper framework, in which meaning derivation by every symbol(s) by situating within a rich ontology. There is room for other modalities, like data structures didn't only include the symbols. Finally, higher level domains can be built as analogical layers on primitive domains. 


\section{REFERENCES:}

[1]. Lenat, Douglas. "Hal's Legacy: 2001's Computer as Dream and Reality. From 2001 to 2001: Common Sense and the Mind of HAL". Cycorp, Inc.

[2]. The Editors of Time-Life Books (1986). Understanding Computers: Artificial

Amsterdam: Time-Life Intelligence.

p. 84. ISBN 0-7054-0915-5.

[3]. Alan Belasco et al. (2004). "Representing Knowledge Gaps Effectively". In: D. Karagiannis, U. Reimer (Eds.): Practical Aspects
ofKnowledge Management, Proceedings of PAKM 2004, Vienna, Austria, December 2-3, 2004. Springer-Verlag, Berlin Heidelberg.

[4]. Jon Curtis et al. (2005). "On the Effective Use of Cyc in a Question Answering System". In: Papers from the IJCAI Workshop on Knowledge and Reasoning for Answering Questions. Edinburgh, Scotland: 2005.

[5]. Chris Deaton et al. (2005). "The Comprehensive Terrorism Knowledge Base in Cyc". In: Proceedings of the 2005 International Conference on Intelligence Analysis, McLean, Virginia, May 2005 